\title{
Towards more resilient electrical networks in urban areas
}

\author{
Anna Palau-Mayo ${ }^{1, *}$, Mikel de Prada ${ }^{1, *}$, and José Luís Domínguez-García ${ }^{1, *}$ \\ ${ }^{1}$ Catalonia Institute for Energy Research, Jardins de les Dones de Negre 1, 2a., 08930 Sant Adrià de \\ Besòs, Barcelona, Spain
}

\begin{abstract}
The requirement of system decarbonisation fixed by the EU 2050 plan is leading to an increased establishment of renewable energy sources. Additionally, the emergence of power electronics and ICT technologies has played a decisive role towards a novel distribution electric grid allowing new monitoring, operation and control. In parallel to the energetic transition, an increasing occurrence of extreme weather events and a reinforced concern on climate change leads to the concept of resilience, which is the capacity to adapt and recover from disruptive events in a coordinated procedure. After a fault event, assuming the objective of the system operator is to minimize the load unsupplied, the present study aims at outlining an early research state on the concept of self-healing through the development of a power flow optimization algorithm within a meshed network. Moreover, the effects of integrating Distributed Energy Resources (DER) in order to increase distribution grid resilience as well as to ensure and secure power supply to the system leads to the clusterization of the power system. With controllable technologies, the on-outaged areas are able to disconnect from the main grid, creating islanded microgrids (MGs) which can work autonomously and consequently, increase grid resilience.
\end{abstract}

\section{Introduction}

The present research is englobed within the project RESCCUE (RESilience to cope with Climate Change in Urban arEas), funded by the European Union's Horizon 2020 Research and Innovation Programme.

Historically, the electrical network has been integrated vertically and centralized, with great generating power plants supplying energy to customers after going across long distances. The current paradigm is forcing the change into a decentralised electrical system due to the appearance of renewable energy sources (RES), energy storage systems (ESS) and electrical vehicles (EV), together with Information and Communication Technologies (ICT). The future energetic model is working towards a distributed energy system with DERs, where the customer will play a central role.

The European Commission has set a target of reaching by 2030 and 2050 a final energy consumption of at least 28\% and 55\%, respectively, coming from RES [1]. Moreover, the increasing occurrence of natural disasters is shedding doubts to the security and supply

\footnotetext{
*Corresponding authors: apalau@irec.cat, mdeprada@irec.cat, jldominguez@irec.cat
} 
continuity of the current power system design [2]. According to the United Nations [3], 66\% of the population will live in urban areas by 2050 and therefore under natural disasters, an Urban Power System (UPS) with an automatic reconfiguration, or self-healing, of the network would enhance resilience under climate change event and consequently, continuity of supply to customers would be ensured [4]. Various self-healing strategies have been defined for the restoration of a power system assuming the latter is formed by is automated and remotely controlled equipment [5-9]. However, these studies also assume a radial network topology, which evidences the need of developing a strategy for meshed urban networks.

In this paper, an early development of a self-healing strategy on load restoration is presented based on the need of increasing resilience. Ongoing results are being tested on the CIGRE benchmark network at medium voltage (MV) [10].

\section{Resilience in urban areas}

As global warming strikes the world, urban electricity demand is expected to increase from $51 \%$ to $58 \%$ between 2006 and 2030 , which evidences the need of having a resilience strategy management in cities [11]. A coordinated and fast response in front of disruptive events in populated areas leads to the concept of resilience, which is the capability to anticipate, prepare, respond and recover from unexpected extreme events, as observed in Figure 1.

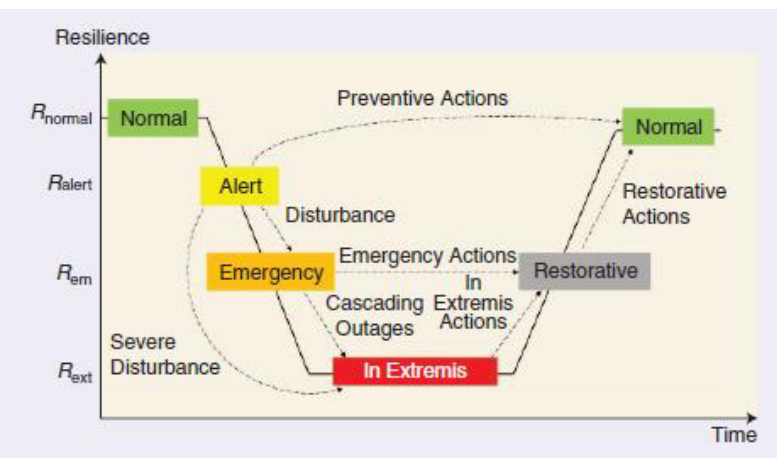

Fig. 1. Resilience curve concept [12]

Building a resilient UPS is based on four key factors: meshed operation, integration of DER units, island mode and automation and communication. A meshed operation of a network consists on more than one feeder supplying the load, and therefore continuity of supply is more probable than in radial operation. Moreover, in an UPS where the majority of the lines are underground and hence the reparation of a possible failure is more complex, meshing the network can also be seen as a preventive measure [13]. Continuity of supply is also enhanced by placing DER units in a UPS since spatial diversification of generation reduces the stress of network components and the designed peak power. Moreover, distributed storage units possess the black-start capability necessary for energizing a system immediately after a fault event [14]. Islanding allows for the restoration of that system in case of a major blackout in the main grid, increasing the flexibility of the system. Island mode operation is dependent on the amount of units with a black-start capability in the system, leading to the need of having the aforementioned storage system units to restart the UPS. Regarding the automation and communication, automated and remotely controlled switches would allow a self-healing of the grid instead of a manual reconfiguration which is present nowadays in the majority of UPS when a contingency occurs. Moreover, phase measurement 
units and local communications become essential in a smart UPS, especially when the system operates as a cluster of microgrids (MGs) [15].

During the planning stage of a power system, static analysis are essential to check the preparedness of the system under unexpected events. To know how resilient the UPS is, contingency analysis are simulated in order to provide information (unsupplied load, violation of network limits, etc.) on possible states of the UPS when a certain equipment is left out of service. In summary, a contingency analysis is essential in determining the current and future potential resilience capacity of an UPS.

\section{Benchmark network at medium voltage}

The CIGRE benchmark at medium voltage (Figure 2) is the network being used for the contingency analysis as it resembles an electric single-line diagram from the perspective of a system operator. Some distributed generation is included in the studied network, as stated in the following paragraph.

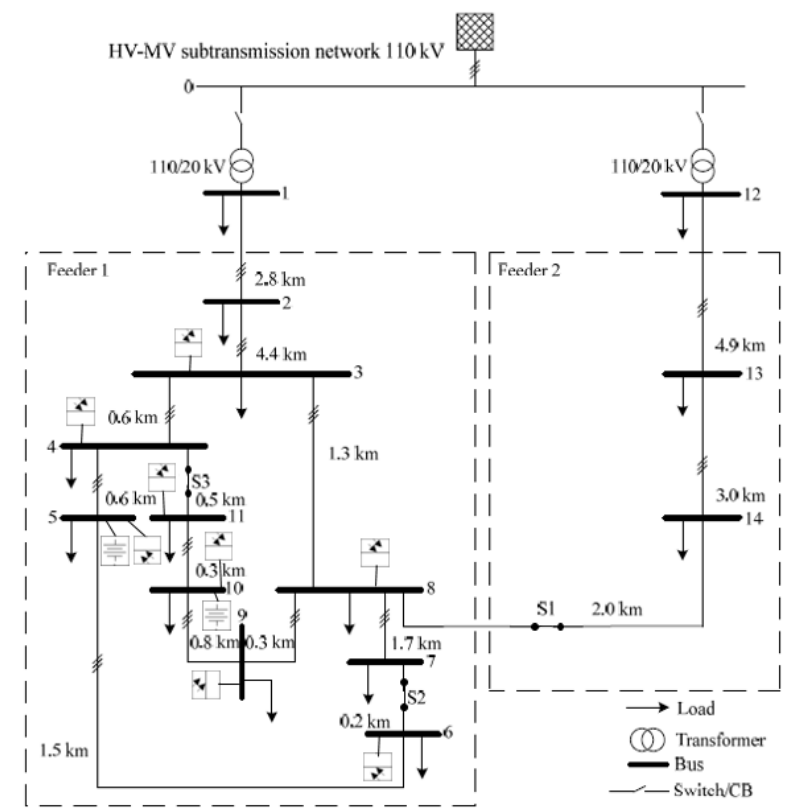

Fig. 2. Test case adapted from the CIGRE European network at medium voltage [10]

As it can be observed in Figure 2, there are three switches (S1, S2 and S3) which are either opened or closed depending on the network topology desired. For the present research, all three switches are closed in order to resemble a real meshed UPS. Both $110 \mathrm{kV} / 20 \mathrm{kV}$ transformers have a rated power of $25 \mathrm{MVA}$ and on-load automatic tap control on the $20 \mathrm{kV}$ side, which is limited within the range of 0.9 p.u. to 1.1 p.u. of the nominal voltage. The HV side of the transformer has a no-load tap position control with a voltage ranging between 0.95 p.u. and 1.05 p.u. Regarding MV loads, there are 34.6 MVA, 34.8MVA, 34.0 MVA, 34.5 MVA and 32.0 MVA of peak load (three-phase and in delta connection) at T0, T1, T2, T3 and T4 respectively. It is remarkable to state that loads at Bus 1 and Bus 12 represent $89 \%$ of the total peak load since these represent additional feeders. To be able to feed all peak loads simultaneously, the network has been designed with a total capacity of 48MVA. Photovoltaic generation is present in bus $3,4,5,6,8,9,10$ and 11 while a battery system is present in both bus 5 and bus 10 . 


\section{Self-healing strategy and results}

After an unexpected outage in a smart UPS, automated system restoration strategies are a key characteristic in rapidly recovering. Knowing that from a system operator perspective, real-time operational strategies are limited to the amount of sensors and how often these send signals, the proposed algorithm plays with a hypothesis. It is assumed that once an outage happens, the system operator gives the on-outage area of the power system 1 hour to recover and that every 15 minutes, measurements are received. This means that there are a total of five time periods.

For the ongoing research, the self-healing strategy is focused in a two-step strategy: blackstart and load restoration Firstly, in the black-start stage, all generators with a black-start capability i.e. being able to turn on without the need of an external supply such as a storage system, must start running in order to re-energize the system in an optimized sequence. Once this first step is fulfilled, the second step of the strategy, which is the point of focus for the present research, is to minimize load unsupplied given certain priorities depending on how critical the infrastructure is. At the same time, for this step, other objectives are the minimization of the line switching actions and the operation of the storage system in order to extend their respective lifecycles. The multi-objective function can is formulated in Equation 1. The binary variable representing the switching between lines has still not been implemented in the current algorithm, so Equation 1 does not reflect this component yet. Let $\alpha$ be the weight parameters, $S_{\text {load }_{n, t}}$ be the apparent power of load in node $n$ at time $t$, be $s b$ the base power, be $x_{\text {load }_{s, t}}$ the status of the load switch $s$ at time $t$, be $w_{\text {load }}$ the load priority at time $t$ and be $x_{b a t_{b, t}}^{c \text { char }}$ and $x_{b a t_{b, t}}^{\text {disc }}$ the status of the battery switch at time $t$.

$$
z=\min \left(\sum_{t \in T}\left(\sum_{s, n \in M A P 3} \alpha \frac{S_{\operatorname{load}_{n, t}}}{s b}\left(1-x_{\operatorname{load}_{s, t}}\right) w_{\operatorname{load}_{s, t}}+\sum_{b \in B}(1-\alpha)\left(x_{\mathrm{bat}_{b, t}}^{\text {char }}+x_{\mathrm{bat}_{b, t}}^{\text {disc }}\right)\right)\right)
$$

The optimization consists of an optimal power flow algorithm in alternate current (AC) with power system restrictions to comply with, such as line thermal limits, state of charge in the battery and voltage limits. The developed algorithm is to be implemented in the CIGRE network after a failure in the network (e.g. line trip) takes place, in order to see how the network automatically reconfigures to fulfil the main objective: minimization of load unsupplied. The battery is forced to end up with a state of charge equal or above $50 \%$. Loads in bus 1, 5, 8, 9 and 14 are considered critical infrastructure and therefore they are prioritized over the rest of the loads to restore. It is important to clarify that the self-healing algorithm only applies to the on-outage area, but since the current studied network is meshed, the selfhealing algorithm applies to the whole power system.

For the present simulation, the $\mathrm{AC}$ algorithm has still not been developed. Therefore, the preliminary results in the current paper are achieved after running an optimal power flow with direct current (DC), which means no losses in the system and no reactive power flow. The deterministic algorithm is implemented in GAMS with the cplex solver. The fault in the system will be forced between Bus 0 to Bus 1 at $\mathrm{T} 0$ and it will be assumed to last during the whole simulation period of 1 hour. In the following Table 1, Table 2 and Table 3, some test results are shown. The pre-fault results refer to the normal-operating conditions while postfault results refer to the system behaviour once the HV/MV transformer connecting Bus 0 and Bus 1 suffers an outage. 
Table 1. Active power discharged by batteries (MW)

\begin{tabular}{|c|c|c|c|c|c|}
\hline & T0 & T1 & T2 & T3 & T4 \\
\hline Battery bus 5 & 0 & 0 & 0.6 & 0 & 0 \\
\hline Battery bus 10 & 0.4 & 0 & 0 & 0 & 0 \\
\hline
\end{tabular}

(a) pre-fault

\begin{tabular}{|c|c|c|c|c|c|}
\hline & $\mathrm{T} 0$ & $\mathrm{~T} 1$ & $\mathrm{~T} 2$ & $\mathrm{~T} 3$ & $\mathrm{~T} 4$ \\
\hline Battery bus 5 & 0.6 & 0 & 0.6 & 0 & 0.2 \\
\hline $\begin{array}{c}\text { Battery bus } \\
10\end{array}$ & 0 & 0.4 & 0 & 0.4 & 0.4 \\
\hline
\end{tabular}

(b) post-fault with optimization

Table 2. Active power charged by batteries (MW)

\begin{tabular}{|c|c|c|c|c|c|}
\hline & T0 & T1 & T2 & T3 & T4 \\
\hline Battery bus 5 & 0 & 0 & 0 & 0.6 & 0 \\
\hline Battery bus 10 & 0 & 0.4 & 0 & 0 & 0 \\
\hline
\end{tabular}

(a) pre-fault

\begin{tabular}{|c|c|c|c|c|c|}
\hline & T0 & T1 & T2 & T3 & T4 \\
\hline Battery bus 5 & 0 & 0.19 & 0 & 0.6 & 0 \\
\hline $\begin{array}{c}\text { Battery bus } \\
10\end{array}$ & 0.4 & 0 & 0.4 & 0 & 0 \\
\hline
\end{tabular}

(b) post-fault with optimization

Table 3. Active power generated by the slack and photovoltaic generators (MW)

\begin{tabular}{|c|c|c|c|c|c|}
\hline & T0 & T1 & T2 & T3 & T4 \\
\hline Slack & 33.2 & 34.3 & 32.2 & 33.3 & 32.1 \\
\hline $\begin{array}{c}\text { PV } \\
\text { bus 3 }\end{array}$ & 0.2 & 0.2 & 0.2 & 0.2 & 0.2 \\
\hline $\begin{array}{c}\text { PV } \\
\text { bus 4 }\end{array}$ & 0.2 & 0.2 & 0.2 & 0.2 & 0.2 \\
\hline $\begin{array}{c}\text { PV } \\
\text { bus 5 }\end{array}$ & 0.3 & 0.3 & 0.3 & 0.3 & 0.3 \\
\hline $\begin{array}{c}\text { PV } \\
\text { bus 6 }\end{array}$ & 0.3 & 0.3 & 0.3 & 0.3 & 0.3 \\
\hline $\begin{array}{c}\text { PV } \\
\text { bus 8 }\end{array}$ & 0.3 & 0.3 & 0.3 & 0.3 & 0.3 \\
\hline $\begin{array}{c}\text { PV } \\
\text { bus 9 }\end{array}$ & 0.3 & 0.3 & 0.3 & 0.3 & 0.3 \\
\hline $\begin{array}{c}\text { PV } \\
\text { bus 10 }\end{array}$ & 0.4 & 0.4 & 0.4 & 0.4 & 0.4 \\
\hline $\begin{array}{c}\text { PV } \\
\text { bus 11 }\end{array}$ & 0.1 & 0.1 & 0.1 & 0.1 & 0.1 \\
\hline
\end{tabular}

(a) pre-fault

\begin{tabular}{|c|c|c|c|c|c|}
\hline & T0 & T1 & T2 & T3 & T4 \\
\hline Slack & 16.1 & 16.2 & 16.1 & 16.7 & 15.1 \\
\hline $\begin{array}{c}\text { PV } \\
\text { bus 3 }\end{array}$ & 0.18 & 0.2 & 0.2 & 0.2 & 0.18 \\
\hline $\begin{array}{c}\text { PV } \\
\text { bus 4 }\end{array}$ & 0.2 & 0.2 & 0.2 & 0.2 & 0.2 \\
\hline $\begin{array}{c}\text { PV } \\
\text { bus 5 }\end{array}$ & 0.3 & 0.3 & 0.3 & 0.3 & 0.3 \\
\hline $\begin{array}{c}\text { PV } \\
\text { bus 6 }\end{array}$ & 0.3 & 0.3 & 0.3 & 0.3 & 0.3 \\
\hline $\begin{array}{c}\text { PV } \\
\text { bus 8 }\end{array}$ & 0.3 & 0.3 & 0.3 & 0.3 & 0.3 \\
\hline $\begin{array}{c}\text { PV } \\
\text { bus 9 }\end{array}$ & 0.3 & 0.3 & 0.3 & 0.3 & 0.3 \\
\hline $\begin{array}{c}\text { PV } \\
\text { bus 10 }\end{array}$ & 0.4 & 0.4 & 0.4 & 0.4 & 0.4 \\
\hline $\begin{array}{c}\text { PV } \\
\text { bus 11 }\end{array}$ & 0.1 & 0.1 & 0.1 & 0.1 & 0.1 \\
\hline
\end{tabular}

(a) post-fault with optimization

During the pre-fault analysis, all load is supplied during all periods of times because there are no contingencies in the network and therefore the capacity of the system is designed to cover up all the demand. On the other hand, during the post-fault simulation, if the optimization described above is implemented, $52 \%, 52 \%, 51 \%, 50 \%$ and $53 \%$ of the load is supplied at each time period $\mathrm{T} 0, \mathrm{~T} 1, \mathrm{~T} 2, \mathrm{~T} 3$ and $\mathrm{T} 4$ respectively. Otherwise, if no optimization is implemented after the fault occurs, a much lower percentage of loads would be supplied, more precisely, $5 \%, 5 \%, 4 \%, 4 \%$ and $3 \%$ respectively. In the following Figure 4 , a plot outlining the percentage of supplied load during each of the scenarios is observed. 


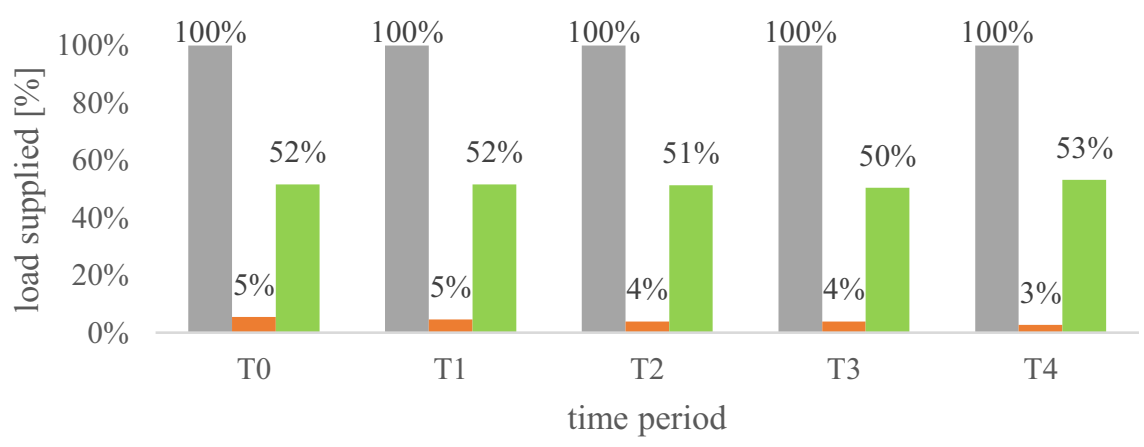

$\square$ pre-fault $\quad$ post-fault with no optimization $\quad$ post-fault with optimization

Fig. 4 Percentage of load supplied in pre-fault and post-fault scenarios [own source]

\section{Conclusion}

The current paper has outlined the need of increasing resilience in a UPS due to the forecasted increase in urban population as well as the factual increase of climate hazard events. The optimization algorithm presented in this paper is at an early research state but even though, results clearly reflect the importance of implementing self-healing techniques under outage events in order to minimize the impact on customers. As future work, an AC optimal power flow in a meshed network is still to be developed. Such algorithm must also include the possibility of clustering the UPS into several smaller power systems in order to decentralise the power generation and increase resilience. Moreover, the optimization algorithm is planned to be scalable to bigger power systems such as a real UPS but the lack of phase measurement units poses a limitation to real life applications.

\section{References}

1. European Commission, "Clean Energy For All Europeans," ed. Brussels: European Commission, 2016.

2. S. Vela, "Identification of Potential Hazards for Urban Strategic Services produced by Extreme Events," public deliverable (RESCCUE Project), 2017.

3. United Nations, Department of Economic and Social Affairs, "World Urbanization Prospects: The 2014 Revision" (ST/ESA/SER.A/352), 2014.

4. T. Huang, "Understanding natural, accidental and malicious threats against secure operation of power systems", ed: Politecnico di Torino - Dipartimento Energia, 2017.

5. C.M. Colson, M.H. Nehrir and R.W. Gunderson, "Distributed multi-agent microgrids: a decentralized approach to resilient power system self-healing," 2011 4th International Symposium on Resilient Control Systems, Boise, ID, 2011, pp. 83-88. doi: 10.1109/ISRCS.2011.6016094

6. A. Golshani, W. Sun, Q. Zhou, Q. P. Zheng and J. Tong, "Two-Stage Adaptive Restoration Decision Support System for a Self-Healing Power Grid," in IEEE Transactions on Industrial Informatics, vol. 13, no. 6, pp. 2802-2812, Dec. 2017. doi: 10.1109/TII.2017.2712147 
7. Z. Wang and J. Wang, "Self-Healing Resilient Distribution Systems Based on Sectionalization Into Microgrids," in IEEE Transactions on Power Systems, vol. 30, no. 6, pp. 3139-3149, Nov. 2015. doi: 10.1109/TPWRS.2015.2389753

8. F. Capitanescu, I. Bilibin and J. Sachau, "Towards optimal post-fault self-healing infuture smart distribution grids", in Int. Conf. Renewable Energies and Power Quality (ICREPQ'13), March 20-22, 2013, Bilbao, Spain, 2013.

9. B. Ansari, M. G. Simoes, A. Soroudi and A. Keane, "Restoration strategy in a selfhealing distribution network with DG and flexible loads," 2016 IEEE 16th International Conference on Environment and Electrical Engineering (EEEIC), Florence, 2016, pp. 1 5. doi: 10.1109/EEEIC.2016.7555776

10. CIGRE, "Benchmark Systems for Network Integration of Renewable and Distributed Energy Resources," (confidential) 2013.

11. International Energy Agency (IEA), "World Energy Outlook," International Energy Agency, Paris, 2008.

12. M. Panteli and P. Mancarella, "Modeling and Evaluating the Resilience of Critical Electrical Power Infrastructure to Extreme Weather Events," in IEEE Systems Journal, vol. 11, no. 3, pp. 1733-1742, Sept. 2017. doi: 10.1109/JSYST.2015.2389272

13. K. Forssén and K. Mäki, "Resilience of finnish electricity distribution networks against extreme weather conditions," in CIRED Workshop 2016, 2016, pp. 1-4.

14. S. Stout, Hotchkiss, E., "Distributed Energy Generation for Climate Resilience," ed. Minnesota: National Adaptation Forum, National Renewable Energy Laboratory (NREL), 2017.

15. H. Liu, X. Chen, K. Yu and Y. Hou, "The Control and Analysis of Self-Healing Urban Power Grid," in IEEE Transactions on Smart Grid, vol. 3, no. 3, pp. 1119-1129, Sept. 2012. doi: 10.1109/TSG.2011.2167525 